\title{
Is Metformin the Answer for Distressed Females with Menstrual Irregularities?
}

Chavi Tejpal ${ }^{1}$, Ishan Poudel ${ }^{2}$, Nusrat Jahan ${ }^{2}$

1. Family Medicine, Department of Research, California Institute of Behavioral Neurosciences and Psychology, Fairfield, USA 2. Internal Medicine, Department of Research, California Institute of Behavioral Neurosciences and Psychology, Fairfield, USA

Corresponding author: Chavi Tejpal, tejpalchavi@gmail.com

\begin{abstract}
This literature review is aimed to determine if metformin alone improves menstrual irregularities in females with the polycystic ovarian syndrome. The current literature review involves females with polycystic ovarian syndrome experiencing menstrual irregularity. The data was collected in PubMed and inclusion criteria included articles published in the past 10 years, articles involving only humans, articles written in the English language and considering women age 19 or more. The number of discovered articles was 1550 after the first search and only 25 articles that met the inclusion criteria were selected after quality assessment. The selected 25 articles that met the inclusion criteria after a review showed evidence for regulating menstrual cycles with metformin therapy alone among females with the polycystic ovarian syndrome. When metformin was used in addition to other pharmacologic agents, there was a more significant restoration of menstrual cycles. Monotherapy with metformin is found to be highly effective in treating menstrual irregularities experienced among patients with the polycystic ovarian syndrome. Greater improvement was noted with the addition of another agent to metformin.
\end{abstract}

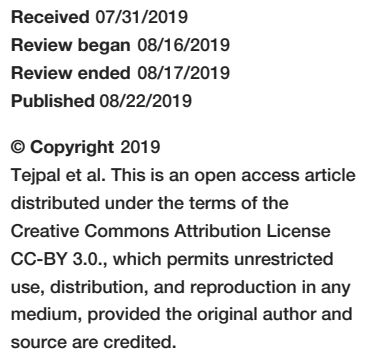

Categories: Family/General Practice, Internal Medicine, Obstetrics/Gynecology Keywords: polycystic ovary syndrome, metformin, menstrual irregularities

\section{Introduction And Background}

The polycystic ovarian syndrome affects women of childbearing age and is a clinical diagnosis. The etiology of the polycystic ovarian syndrome is unknown but may be attributed to insulin resistance. Patients frequently present with symptoms such as menstrual irregularities, androgen excess, and polycystic ovaries. While androgen excess often presents with acne and hirsutism, menstrual irregularities often lead to infertility. Treating menstrual irregularities is a crucial component to the polycystic ovarian syndrome. Menstrual irregularities caused by polycystic ovaries leads to infertility, resulting in distress among women trying to conceive [1-3].

Hormonal pharmacologic therapies such as oral contraceptives have been used for the treatment of menstrual irregularities in polycystic ovarian syndrome. In addition to hormone therapy, metformin was used to treat irregular menses in females with a history of oligo-menorrhea or amenorrhea. The exact mechanism of metformin in regulating menstrual cycle is still unknown. However, it may be attributed to insulin sensitization thereby targeting the insulin resistance caused by androgen excess [1-3]. This literature review is aimed to determine the effectiveness of metformin in regulating menstrual cycles among females with the polycystic ovarian syndrome.

\section{Review \\ Method}

The data was collected from PubMed using regular keywords "polycystic ovary" and "metformin.” The following inclusion criteria were applied in the following order - literature published within 10 years, articles involving only human subjects, articles published only in English language and the age of the subjects 19 years or more. The collection of the articles for the study was done ethically.

\section{Results}

After using regular keywords on PubMed, a total of 1550 articles were identified. Among the 1550 articles, 347 were obtained with the application of filters. Table 1 shows the total number of articles obtained in order after applying inclusion criteria in PubMed. 


\title{
Cureus
}

\begin{tabular}{|l|l|}
\hline Articles & Results \\
\hline Total articles found & 1550 \\
\hline After inclusion criteria & \\
10 years & 890 \\
Humans & 706 \\
English language & 658 \\
\hline Age 19 or more & 347 \\
\hline
\end{tabular}

TABLE 1: Keywords search after applying inclusion criteria in PubMed

Of the 347 articles obtained, each article was individually reviewed based on the abstract content. Three hundred and twenty-two articles were not selected for review due to one or more of the following reasons:

\author{
- lack of disease of interest; \\ - lack of menstrual irregularities; \\ - lack of metformin use for menstrual irregularity; \\ - studies with no possible derivation of information with quality assessment.
}

The number of articles obtained after removal of 322 articles was 25 , among which 16 were free abstracts available online for review and nine were free with full text available online for review. Of the articles included in the data, there were 21 randomized controlled trials, two literature reviews, and one systemic review.

In addition to metformin, other drugs used as comparators in the randomized clinical trials were clomiphene citrate, oral contraceptives, ethinylestradiol, desogestrel, simvastatin, rosiglitazone, orlistat, nacetylcysteine, letrozole, calcium \& vitamin D, pioglitazone, saxagliptin, spironolactone, flutamide, lipoic acid, monacolin K and myo-inositol. Non-pharmacologic therapies used in clinical trials were lifestyle modifications such as caloric restriction and exercise [3-12].

The 25 selected articles showed evidence for metformin therapy to be efficacious in regulating menstrual cycles of females with the polycystic ovarian syndrome. When metformin was used with other pharmacologic agents, there was a recovery of menstrual cycles as well. Of the articles collected, the randomized clinical trials involving metformin showed improvement in menstrual cycle irregularity with metformin [3, 10-17]. Table 2 shows the analysis of various parameters seen in the randomized clinical trials. 


\begin{tabular}{|c|c|c|c|c|c|c|c|c|c|}
\hline \multicolumn{10}{|c|}{ d Controlled } \\
\hline & & Metformin & & & Results & & & & \\
\hline 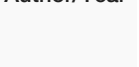 & opraction & $\begin{array}{l}\text { if } \\
\text { applicable) }\end{array}$ & mo & (Months) & Menses & LH/FSH & Weight & Testosterone & Other \\
\hline $\begin{array}{l}\text { Seyam et } \\
\text { al. } 2018 \text { [16] }\end{array}$ & 65 & $\begin{array}{l}500 \mathrm{mg} \text { three } \\
\text { times daily }\end{array}$ & $\begin{array}{l}\text { Group A } \\
\text { simvastatin + } \\
\text { metformin, group } \\
\text { B simvastatin, } \\
\text { group C metformin }\end{array}$ & 12 & Improved & $\begin{array}{l}\text { Decrease } \\
\text { serum LH } \\
\text { level }\end{array}$ & Decrease BMI & Decrease & $\begin{array}{l}\text { Decrease of total } \\
\text { cholesterol, low-densit } \\
\text { lipoprotein, triglyceridt } \\
\text { increase in high-densit } \\
\text { lipoprotein decreased } \\
\text { hirsutism, acne, ovaria } \\
\text { volume }\end{array}$ \\
\hline $\begin{array}{l}\text { Alpañés et } \\
\text { al. } 2017 \text { [3] }\end{array}$ & 22 & 850 mg BID & $\begin{array}{l}\text { Group with } \\
\text { combined oral } \\
\text { contraceptive } \\
\text { (COC) plus } \\
\text { spironolactone } \\
\text { versus metformin }\end{array}$ & 12 & $\begin{array}{l}\text { Menstrual } \\
\text { dysfunction } \\
\text { was less } \\
\text { frequent with } \\
\text { cOC plus } \\
\text { spironolactone }\end{array}$ & N/A & N/A & $\begin{array}{l}\text { Total } \\
\text { testosterone, } \\
\text { free } \\
\text { testosterone }\end{array}$ & $\begin{array}{l}\text { COC + spironolactone } \\
\text { showed more decreas } \\
\text { in hirsutism, } \\
\text { androstenedione, } \\
\text { dehydroepiandrostero } \\
\text { sulphate }\end{array}$ \\
\hline $\begin{array}{l}\text { Tagliaferri } \\
\text { et al. } 2017 \\
\text { [11] }\end{array}$ & 34 & 850 mg BID & $\begin{array}{l}\text { Group } \\
\text { metformin versus } \\
\text { myoinositol }\end{array}$ & 6 & $\begin{array}{l}\text { Improved } \\
\text { menstrual } \\
\text { pattern with } \\
\text { metformin }\end{array}$ & $\begin{array}{l}\text { Metformin } \\
\text { showed } \\
\text { LH } \\
\text { significant } \\
\text { decreased }\end{array}$ & $\begin{array}{l}\text { Metformin } \\
\text { showed a } \\
\text { decrease in } \\
\text { weight }\end{array}$ & N/A & $\begin{array}{l}\text { Metformin showed a } \\
\text { decrease in estradiol } \\
\text { levels and androgens }\end{array}$ \\
\hline $\begin{array}{l}\text { Elkind- } \\
\text { Hirsch et al. } \\
2017 \text { [17] }\end{array}$ & 3 & $2,000 \mathrm{mg}$ & $\begin{array}{l}\text { Combined } \\
\text { saxagliptin } 2,000 \\
\text { mg-metformin } 5 \\
\text { mg, saxagliptin } \\
5 \mathrm{mg} \text {, or metformin } \\
2,000 \mathrm{mg}\end{array}$ & 4 & $\begin{array}{l}\text { Saxagliptin- } \\
\text { metformin } \\
\text { greatly } \\
\text { improved the } \\
\text { menstrual } \\
\text { irregularity }\end{array}$ & N/A & $\begin{array}{l}\text { BMI, waist/height } \\
\text { ratio and waist } \\
\text { circumference } \\
\text { decreased in all } \\
\text { groups }\end{array}$ & N/A & $\begin{array}{l}\text { Significant decrease ir } \\
\text { triglyceride, TG/HDL } \\
\text { cholesterol ratio, and } \\
\text { blood glucose level in } \\
\text { the saxagliptin- } \\
\text { metformin and } \\
\text { saxagliptin groups onl: }\end{array}$ \\
\hline $\begin{array}{l}\text { Mazza et al. } \\
2014 \text { [12] }\end{array}$ & 28 & 1700 mg & $\begin{array}{l}\text { Group A } \\
\text { metformin (1700 } \\
\mathrm{mg} / \text { day), group B } \\
\text { with } \\
\text { metformin (1700 } \\
\mathrm{mg} / \text { day) + } \\
\text { spironolactone ( } 25 \\
\mathrm{mg} / \text { day) }\end{array}$ & N/A & $\begin{array}{l}\text { Menses } \\
\text { improved } \\
\text { more in } \\
\text { metformin } \\
1700 \text { mg a day }\end{array}$ & N/A & N/A & $\begin{array}{l}\text { Significant } \\
\text { decrease in } \\
\text { testosterone } \\
\text { in both } \\
\text { groups }\end{array}$ & $\begin{array}{l}\text { Significant decrease ir } \\
\text { group } A \text { and B of } \\
\text { androstenedione and } \\
\text { hirsutism }\end{array}$ \\
\hline $\begin{array}{l}\text { Firouzabadi } \\
\text { et al. } 2012 \\
\text { [13] }\end{array}$ & 50 & 1500 mg/day & $\begin{array}{l}\text { Group I } \\
\text { metformin } 1500 \mathrm{mg} \\
\text { a day, group II } \\
\text { metformin } 1500 \mathrm{mg} \\
\text { a day + calcium } \\
1000 \mathrm{mg} \text { a day + } \\
\text { vitamin D } \\
100000 \text { IU per } \\
\text { month }\end{array}$ & 6 & $\begin{array}{l}\text { Menses } \\
\text { improved } \\
\text { more in } \\
\text { metformin } \\
1500 \mathrm{mg} \text { a day }\end{array}$ & N/A & $\begin{array}{l}\text { Significant } \\
\text { decrease in BMI } \\
\text { in group II }\end{array}$ & N/A & $\begin{array}{l}\text { Follicle maturation, an } \\
\text { infertility improved } \\
\text { group II > group I }\end{array}$ \\
\hline
\end{tabular}

\section{TABLE 2: Analysis of various parameters}

mg - milligram; LH - luteinizing hormone; FSH - follicle-stimulating hormone; BID - two times a day; BMI - body mass index; COC - combined oral contraceptives; N/A - not available; TG - triglyceride; HDL - high-density lipoprotein

Irrespective of the dosage, it is observed that even at a $500 \mathrm{mg}$ dosage, metformin therapy showed improvement in regulating menstrual cycles. In addition, metformin also showed a decrease of hormone levels such as luteinizing hormone (LH), luteinizing hormone/follicle-stimulating hormone (LH/FSH) ratio, testosterone, estradiol, dehydroepiandrosterone sulfate (DHEA-S) and progesterone. Furthermore, metformin was effective in weight reduction and hirsutism [15, 18-19].

Table 3 below illustrates the effects seen with metformin monotherapy at various doses in the following randomized clinical trials in patients with polycystic ovarian syndrome. 


\section{Cureus}

\begin{tabular}{|c|c|c|c|c|c|c|c|c|}
\hline \multicolumn{9}{|c|}{ Randomized Control Trial with metformin only } \\
\hline \multirow{2}{*}{$\begin{array}{l}\text { Author/ } \\
\text { Year }\end{array}$} & \multirow{2}{*}{ Population } & \multirow{2}{*}{ Dose (mg) } & \multirow{2}{*}{$\begin{array}{l}\text { Length of } \\
\text { Study } \\
\text { (Months) }\end{array}$} & \multicolumn{5}{|l|}{ Results } \\
\hline & & & & Menses & LH/FSH & Weight & Testosterone & Other \\
\hline $\begin{array}{l}\text { Fulgnesu } \\
\text { et al. } \\
2012 \text { [15] }\end{array}$ & 201 & $\begin{array}{l}500 \mathrm{mg} \mathrm{BID}, 500 \mathrm{mg} \text { TID, } \\
850 \mathrm{mg} \text { BID }\end{array}$ & 6 & $\begin{array}{l}\text { Increase of } \\
\text { more than } \\
\text { two } \\
\text { menstrual } \\
\text { cycles/year }\end{array}$ & $\mathrm{N} / \mathrm{A}$ & $\mathrm{N} / \mathrm{A}$ & $\begin{array}{l}\text { Greater } \\
\text { reduction in } \\
\text { plasma } \\
\text { testosterone } \\
\text { levels who had } \\
\text { a higher basal } \\
\text { BMI }\end{array}$ & $\mathrm{N} / \mathrm{A}$ \\
\hline $\begin{array}{l}\text { Yang et } \\
\text { al. } 2018 \\
{[18]}\end{array}$ & 119 & $\begin{array}{l}\text { The first month } 500 \mathrm{mg} \\
\text { once; the second month } \\
1000 \mathrm{mg} \text { once; third month } \\
\text { \& onwards } 1500 \mathrm{mg} \text { once }\end{array}$ & 24 & $\begin{array}{l}\text { Increased } \\
\text { frequency }\end{array}$ & $\begin{array}{l}\text { Decreased } \\
\text { LH }\end{array}$ & $\begin{array}{l}\text { Decreased } \\
\text { BMI }\end{array}$ & Decreased & $\mathrm{N} / \mathrm{A}$ \\
\hline $\begin{array}{l}\text { Velija- } \\
\text { Ašimi et } \\
\text { al. } 2013 \\
{[19]}\end{array}$ & 100 & 1000-1500 mg BID/TID & 12 & Restored & $\begin{array}{l}\text { Decreased } \\
\text { LH, } \\
\text { LH/FSH }\end{array}$ & $\begin{array}{l}\text { Reduced } \\
\text { weight, waist } \\
\text { circumference, } \\
\text { BMI }\end{array}$ & Decreased & $\begin{array}{l}\text { Decrease } \\
\text { estradiol, } \\
\text { CRP, DHEA-S } \\
\text { and } \\
\text { progesterone, } \\
\text { hirsutism }\end{array}$ \\
\hline
\end{tabular}

\section{TABLE 3: Randomized clinical trials involving monotherapy of metformin at various doses}

mg - milligram; LH - luteinizing hormone; FSH - follicle-stimulating hormone; BID - two times a day; TID - three times a day; BMI - body mass index; CRP - c-reactive protein; DHEA-S - dehydroepiandrosterone sulfate; N/A - not available

\section{Conclusions}

The objective of the current literature review is to determine the effectiveness of metformin in regulating menstrual cycles among women diagnosed with the polycystic ovarian syndrome experiencing irregular menses. The use of metformin has demonstrated an improvement in regulating menses significantly with a greater improvement when metformin is used as an adjunctive to another pharmacologic agent. It is to be determined whether these patients will require lifelong metformin therapy or if insulin sensitization can be achieved at a specific number of doses required thereby leaving room for speculation if metformin can be halted after a certain number of doses. One wonders if metformin's effect at regulating menses is tied with insulin sensitization and if serum testosterone plays a role.

\section{Additional Information}

\section{Disclosures}

Conflicts of interest: In compliance with the ICMJE uniform disclosure form, all authors declare the following: Payment/services info: All authors have declared that no financial support was received from any organization for the submitted work. Financial relationships: All authors have declared that they have no financial relationships at present or within the previous three years with any organizations that might have an interest in the submitted work. Other relationships: All authors have declared that there are no other relationships or activities that could appear to have influenced the submitted work.

\section{References}

1. Al Khalifah RA, Florez ID, Dennis B, Thabane L, Bassilious E: Metformin or oral contraceptives for adolescents with polycystic ovarian syndrome: a meta-analysis. J Pediatr. 2016, 137:e20154089. 10.1542/peds.2015-4089

2. Legro RS, Arslanian SA, Ehrmann DA et al.: Diagnosis and treatment of polycystic ovary syndrome: an endocrine society clinical practice guideline. J Clin Endocrinol Metab. 2013, 98:4565-4592. 10.1210/jc.20132350

3. Alpañés M, Álvarez-Blasco F, Fernández-Durán E, Luque-Ramírez M, Escobar-Morreale HF: Combined oral contraceptives plus spironolactone compared with metformin in women with polycystic ovary syndrome: a one-year randomized clinical trial. Eur J Endocrinol. 2017, 177:399-408. 10.1530/EJE-17-0516

4. Elkind-Hirsch KE, Paterson MS, Seidemann EL, Gutowski HC: Short-term therapy with combination dipeptidyl peptidase-4 inhibitor saxagliptin/metformin extended release (XR) is superior to saxagliptin or metformin XR monotherapy in prediabetic women with polycystic ovary syndrome: a single-blind, randomized, pilot study. Fertil Steril. Issue 1, 107:253-260. 10.1016/i.fertnstert.2016.09.023

5. Fruzzetti F, Perini D, Russo M, Bucci F, Gadducci A: Comparison of two insulin sensitizers, metformin and myo-inositol, in women with polycystic ovary syndrome. Gynecol Endocrinol. 2017, 33:39-42. 
6. Seyam E, Hefzy E: Long-term effects of combined simvastatin and metformin treatment on the clinical abnormalities and ovulation dysfunction in single young women with polycystic ovary syndrome. Gynecol Endocrinol. 2018, 34:1073-1080. 10.1080/09513590.2018.1490405

7. Altinok ML, Ravn P, Andersen M, Glintborg D: Effect of 12-month treatment with metformin and/or oral contraceptives on health-related quality of life in polycystic ovary syndrome. Gynecol Endocrinol. 2018, 10:859-863. 10.1080/09513590.2018.1460343

8. Yang P-K, Hsu C-Y, Chen M-J, et al.: The efficacy of 24-month metformin for improving menses, hormones, and metabolic profiles in polycystic ovary syndrome. J Clin Endocrinol Metab. 2018, 103:890-899. 10.1210/jc.2017-01739

9. Kazerooni T, Shojaei-Baghini A, Dehbashi S, Asadi N, Ghaffarpasand F, Kazeroonic Y: Effects of metformin plus simvastatin on polycystic ovary syndrome: a prospective, randomized, double-blind, placebocontrolled study. Fertil Steril. 2010, Volume 94:2208-2213. 10.1016/j.fertnstert.2009.11.045

10. Figurová J, Dravecká I, Petríková J, Javorský M, Lazúrová I: The effect of alfacalcidiol and metformin on metabolic disturbances in women with polycystic ovary syndrome. Horm Mol Biol Clin Investig. 2017, 29:8591. 10.1515/hmbci-2016-0039

11. Tagliaferri V, Romualdi D, Immediata V, et al.: Metformin vs myoinositol: which is better in obese polycystic ovary syndrome patients? A randomized controlled crossover study. Clin Endocrinol. 2017, 86:725-730. 10.1111/cen.13304

12. Mazza A, Fruci B, Guzzi P, et al.: In PCOS patients the addition of low-dose spironolactone induces a more marked reduction of clinical and biochemical hyperandrogenism than metformin alone. Nutr Metab Cardiovasc Dis. 2014, 24:132-139. 10.1016/j.numecd.2013.04.016

13. Firouzabadi RD, Aflatoonian A, Modarresi S, Sekhavat L, Taheri SM: Therapeutic effects of calcium \& vitamin D supplementation in women with PCOS. Complement Ther Clin Pract. 2012, 18:85-88. 10.1016/j.ctcp.2012.01.005

14. Oner G, Muderris II: Clinical, endocrine and metabolic effects of metformin vs N-acetyl-cysteine in women with polycystic ovary syndrome. Eur J Obstet Gynecol Reprod Biol. 2011, 159:127-131. 10.1016/j.ejogrb.2011.07.005

15. Fulghesu AM, Romualdi D, Di Florio C, et al.: Is there a dose-response relationship of metformin treatment in patients with polycystic ovary syndrome? Results from a multicentric study. Hum Reprod. 2012, 27:30573066. 10.1093/humrep/des262

16. Seyam E, Hefzy E: Long-term effects of combined simvastatin and metformin treatment on the clinical abnormalities and ovulation dysfunction in single young women with polycystic ovary syndrome. Gynecol Endocrinol. 2018, 34:1073-1080. 10.1080/09513590.2018.1490405

17. Elkind-Hirsch KE, Paterson MS, Seidemann EL, Gutowski HC: Short-term therapy with combination dipeptidyl peptidase-4 inhibitor saxagliptin/metformin extended release (XR) is superior to saxagliptin or metformin XR monotherapy in prediabetic women with polycystic ovary syndrome: a single-blind, randomized, pilot study. Fertil Steril. 2017, 107:253-260. 10.1016/j.fertnstert.2016.09.023

18. Yang PK, Hsu CY, Chen MJ, et al.: The efficacy of 24-month metformin for improving menses, hormones, and metabolic profiles in polycystic ovary syndrome. J Clin Endocrinol Metab. 2018, 103:890-899. 10.1210/jc.2017-01739

19. Velija-Ašimi Z: Evaluation of endocrine changes in women with the polycystic ovary syndrome during metformin treatment. Bosn J Basic Med Sci. 2013, 13:180-185. 10.17305/bjbms.2013.2359 